\title{
Spectral statistics in noninteracting many-particle systems
}

\author{
L. Muñoz, ${ }^{1}$ E. Faleiro, ${ }^{2}$ R. A. Molina, ${ }^{3}$ A. Relaño, ${ }^{1}$ and J. Retamosa ${ }^{1}$ \\ ${ }^{1}$ Departamento de Física Atómica, Molecular y Nuclear, Universidad Complutense de Madrid, E-28040 Madrid, Spain \\ ${ }^{2}$ Departamento de Física Aplicada, E.U.I.T. Industrial, Universidad Politécnica de Madrid, E-28012 Madrid, Spain \\ ${ }^{3}$ Max-Planck-Institut für Physik Komplexer Systeme, Nöthnitzer Strasse 38, D-01187 Dresden, Germany
}

(Received 31 March 2005; revised manuscript received 12 January 2006; published 2 March 2006)

\begin{abstract}
It is widely accepted that the statistical properties of energy level spectra provide an essential characterization of quantum chaos. Indeed, the spectral fluctuations of many different systems like quantum billiards, atoms, or atomic nuclei have been studied. However, noninteracting many-body systems have received little attention, since it is assumed that they must exhibit Poisson-like fluctuations. Apart from a heuristic argument of Bloch, there are neither systematic numerical calculations nor a rigorous derivation of this fact. Here we present a rigorous study of the spectral fluctuations of noninteracting identical particles moving freely in a mean field emphasizing the evolution with the number of particles $N$ as well as with the energy. Our results are conclusive. For $N \geqslant 2$ the spectra of these systems exhibit Poisson fluctuations provided that we consider sufficiently high excitation energies. Nevertheless, when the mean field is chaotic there exists a critical energy scale $L_{c}$; beyond this scale, the fluctuations deviate from the Poisson statistics as a reminiscence of the statistical properties of the mean field.
\end{abstract}

DOI: 10.1103/PhysRevE.73.036202

PACS number(s): 05.45.Mt, 05.45.Pq, 24.60.-k

\section{INTRODUCTION}

Although the concept of quantum chaos [1] has no unique precise definition as yet, it is well known that there is a relationship between energy level fluctuation properties of a quantum system and the dynamics of its classical analog. Classically integrable systems give rise to uncorrelated adjacent energy levels that are well described by Poisson statistics [2]. In contrast, spectral fluctuations of a quantum system whose classical limit is fully chaotic (ergodic) show a strong repulsion between energy levels and follow the predictions of random matrix theory (RMT) [3,4]. Moreover, quantum systems without classical limit are, in practice, assumed to be chaotic when their fluctuations coincide with RMT predictions.

In this work we analyze the energy spectral fluctuations of $N$ noninteracting identical particles (NIPs) moving freely in a mean field (MF). This subject has received little attention in the scientific literature since in 1969 Bloch argued that the spectra of noninteracting nucleons always display Poisson fluctuations, even if the MF is chaotic [5]. Because of its simplicity, Bloch's argument is valid for noninteracting fermion (NIF) systems as well as for noninteracting boson (NIB) systems. Moreover, in many-body (MB) systems the symmetries very often lead to a regular MF; this is the case of Galilean invariance in atoms or atomic nuclei. For this reason, quantum chaos investigation in MB systems has very often focused on different aspects of the transition from a regular single-particle Hamiltonian to a chaotic Hamiltonian with two-body (or, in more general cases, few-body) interactions [6]. Semiclassical theory has also been used to study MB systems. In spite of the problems raised by the symmetrization postulate and the existence of continuous symmetries (periodic orbits of the full phase space occur in continuous families), the periodic-orbit theory can be adapted to study these types of systems $[7,8]$. Although there have been a few interesting applications to few-body Coulomb systems
[9] and to noninteracting particles [10], most effort has gone into developing semiclassical descriptions of the singleparticle (SP) dynamics in an appropriate MF.

Bloch's original argument states that two successive energy levels of the many-body spectrum, $E_{n}$ and $E_{n+1}$, are sums of energies of very different parts of the SP spectrum and therefore their variations will not be correlated. Clearly, the argument is qualitative and to some extent vague; it does not precise whether NIP systems always follow the Poisson statistics or if certain conditions must be fulfilled. Bloch's argument seems to be plausible for a large number of particles and especially at high excitation energy. However, in the low-energy domain of the MB spectrum the levels are sums of energies pertaining to a small interval of the SP spectrum, and therefore is not clear to what extent we can consider them as uncorrelated. To the best of our knowledge, there are no systematic analytical or numerical calculation trying to address these questions. This is, perhaps, the reason why there is some controversy about the validity of the argument when the number of particles is small: some authors consider Bloch's argument valid already for $N \geqslant 2$ (see, for instance, Ref. [11]), some others assume that is valid when $N \gg 1$ [12], and others claim that it may be to some extent true when $N=2$ [13]. None of them consider the dependence of the spectral fluctuations of the MB spectrum on the energy.

Very often, the spectral fluctuations of MB systems are studied by means of random Hamiltonians with $k$-body interactions defined in finite spaces. Given a set of $m$ SP states, the Hamiltonians are written within the framework of second quantization, and then propagated to the $N$-particle Hilbert space by using the direct-product structure of these spaces [6]. When $k<N$ numerical simulations are the rule, but a few analytical results are known in the so called dilute limit, which corresponds to $(N, m) \rightarrow \infty, N / m \rightarrow 0$ [14]. In this context, using the binary correlation method it has been recently 
claimed that spectral fluctuations become Poissonian when $k \ll N$ [13]. This could be considered as an analytical proof of Bloch's qualitative argument for $N \gg 1$. Unfortunately, it seems that this result is incorrect; the binary correlation approximation is not appropriate even in the simplest case $(k$ $=1$ ), since it involves formal manipulations of divergent series [12].

All these antecedents suggest that there is a common intuition about what should happen with fluctuations in noninteracting particle systems. However, there is neither agreement about the validity of Bloch's argument for small number of particles or low energies, nor systematic numerical or analytical studies to address these questions.

The main purpose of this work is to study the evolution of the spectral fluctuations of NIP systems with the number of particles and the excitation energy. We tackle the problem by performing a rigorous numerical study where there are infinite SP energy levels accessible to each particle. This is an advantage of noninteracting particle systems, which allows us to deal with infinite spaces in contrast to interacting systems where finite spaces must be used.

The paper is organized as follows. Section II describes the Hamiltonian and the MB spectrum of noninteracting particle systems. We explain in Sec. III how the statistical analysis is performed; we introduce the basic statistics and stress the relevance of the unfolding procedure. Here, the derivation of the MB level density is outlined in detail. The main results are presented and discussed in Secs. IV and V. The former, devoted to fermion systems, is divided in two parts, where we study the evolution of the spectral fluctuations with the number of particles and with the excitation energy. The results obtained for boson systems are displayed in Sec. V. Section VI outlines an analytical calculation for $N=2$ systems. The last section (Sec. VII) contains a brief summary and quotes the main conclusions. Finally, the Appendix treats some secondary mathematical details of the integrals appearing in Sec. VI.

\section{THE MODEL}

To be specific, consider $N$ noninteracting identical particles moving freely in a certain MF $\hat{h}$. For simplicity, we shall consider spinless particles, and thus the (one-body) Hamiltonian of the system is given by

$$
\hat{\mathcal{H}}=\sum_{i=1}^{N} \hat{h}\left(\boldsymbol{p}_{i}, \boldsymbol{q}_{i}\right)=\sum_{i=1}^{N}\left(\frac{\boldsymbol{p}_{i}^{2}}{2 \mu}+\hat{\mathcal{V}}\left(\boldsymbol{q}_{i}\right)\right),
$$

where $\left(\boldsymbol{p}_{i}, \boldsymbol{q}_{i}\right)$ are the dynamical variables of the $i$ th particle with mass $\mu$. The Hamiltonian can also be written in terms of the eigenvalues and eigenstates $\left\{e_{i},|i\rangle \mid i \in \mathbb{N}\right\}$ of the SP Hamiltonian $\hat{h}\left(\boldsymbol{p}_{i}, \boldsymbol{q}_{i}\right)$ as

$$
\hat{\mathcal{H}}=\sum_{i \in \mathbb{N}} e_{i}|i\rangle\langle i|,
$$

where the number of SP levels accessible to each particle is infinite. Below we explain how to obtain exact and complete sequences of the actual MB level spectrum with the desired length. We would like to stress that this procedure is completely different from performing the calculations in a finite space and taking the limit $m \rightarrow \infty$ at the end.

The energy levels of the MB system can be calculated as

$$
E_{I}=\sum_{i_{j} \in I} e_{i_{j}}
$$

where $I=\left\{i_{1} \leqslant i_{2} \leqslant \cdots \leqslant i_{N}\right\}$ is the ordered sequence of SP states occupied in the (anti)symmetrized state $|I\rangle$, defined as

$$
|I\rangle=C_{l} \hat{\mathcal{U}}\left|i_{1}\right\rangle \otimes\left|i_{2}\right\rangle \otimes \cdots \otimes\left|i_{N}\right\rangle,
$$

where $\otimes$ stands for the direct product, $C_{I}$ is a state-dependent normalization coefficient, different for fermions and bosons, and $\hat{\mathcal{U}}$ is the so called (anti)symmetrizer. Their expressions are

$$
C_{I}= \begin{cases}1 & \text { for fermions }, \\ \frac{1}{\sqrt{\prod n_{j} !}} & \text { for bosons }\end{cases}
$$

where $n_{j}$ is the number of times that the index $j$ appears in $I$. On the other hand

$$
\hat{\mathcal{U}}=\frac{1}{\sqrt{N !}} \sum_{P} \phi^{t} \hat{\mathcal{P}} .
$$

Here, $\Sigma_{P}$ runs over all the permutations of $N$ symbols, $\phi$ $=-1(+1)$ for fermions (bosons), $t_{p}$ is the number of basic transpositions associated with permutation $P$, and $\hat{P}$ is the permutation operator. For many-fermion systems, the antisymmetrizer guarantees that only configurations with $N$ different SP levels contribute.

Before proceeding further, it is worth making several remarks.

(i) In practice, one cannot deal with the infinite SP spectrum, but only with a finite set of energy levels. Let us assume that we generate all the possible configurations of $N$ identical particles in the valence space made of the first $m$ SP states. These states are usually named model states (or configurations). One of the most serious shortcomings of the valence description of $\mathrm{MB}$ systems is the existence of intruder states. These configurations, lying inside the energy window spanned by the model states, involve SP levels that do not pertain to the valence space. There exists a threshold energy $E_{\max }$ where these configurations start to appear, and any sequence including model states beyond this energy is always incomplete. The great advantage of noninteracting systems is that the threshold energy can be calculated easily as $E_{\text {max }}=E_{1}^{(N-1)}+e_{m+1}$, where $E_{1}^{(N-1)}$ is the ground state energy of the system with $N-1$ particles. Thus, discarding every configuration whose energy is greater or equal to $E_{\max }$ we can generate the exact many-body spectrum up to that energy. Notice that for interacting systems the problem is far more complicated.

(ii) We assume that the statistical properties of the SP spectrum are those of an appropriate matrix ensemble. We also assume that the eigenvalue sequence is stationary and that its average density is equal to one. Of course, these 
might be crude assumptions of the model because the statistical properties of low-lying SP energy levels may not be well described by a RMT approach. Moreover, we ignore some features that may appear in physical systems, like the smooth variation of the level density with the energy, the existence of large energy gaps, energy bands, etc. In this simple version the results can apply when only the properties near the Fermi level are of interest or in two-dimensional problems, like quantum dots, where the particles are trapped in a certain closure.

(iii) This type of systems (and MB systems in general) are most often not integrable. According to the widely accepted criterion of integrability in quantum mechanics [15-19], NIP systems have $N \times d$ degrees of freedom ( $d$ being the spatial dimensions) and, in general, only the energies of each particle would be integrals of motion. In spite of their simplicity, Eqs. (3) and (4) do not define a solvable problem. They give all the exact eigenvalues and eigenstates of the systems, provided that we know all the SP energies $\left\{e_{i}\right\}$. However, these are not usually known analytically and we must calculate numerically a large enough sequence of SP energies to perform the statistical analysis.

(iv) It is sometimes assumed that in many-body systems a clear semiclassical picture arises in the MF approximation; then, the classical limit of the SP states is regular or chaotic depending on the symmetries of the MF potential. However, the MB spectrum is not only defined by the MF potential, but also by the direct product structure of the Hilbert space and by the fermionic or bosonic character of the particles. Moreover, in quantum mechanics identical particles are truly indistinguishable; in particular, we can neither label the different particles of the system nor follow their trajectories because this would entail a position measurement disturbing deeply the system. As a consequence, it is intrinsically impossible to find a classical analog, except for very dilute systems where the overlap among particle wave packets is negligible.

\section{STATISTICAL ANALYSIS}

The task now is to characterize the spectral fluctuations of noninteracting systems, with $N=1,2, \ldots$ particles occupying the infinite SP energy levels of a MF. As commented above, the SP spectra are modeled using random matrix ensembles with different symmetries, like the Gaussian orthogonal ensemble (GOE) and the Gaussian unitary ensemble (GUE). Using the method explained in the previous section we have generated in all the cases complete sequences of about $M$ $=10^{6}$ levels.

\section{A. The MB level density}

Prior to the statistical analysis, the spectrum must be transformed. It is generally accepted that the level density $g(E)$ can be separated into a smooth part $\bar{g}(E)$ that defines the main trend of the level density and a fluctuating part $\widetilde{g}(E)$. The fluctuation amplitudes of the latter are modulated by $\bar{g}(E)$; therefore, to compare the statistical properties of different systems or different parts of the same spectrum, the main trend defined by $\bar{g}(E)$ must be removed. The procedure by which $\bar{g}(E)$ is removed, called unfolding, consists in locally mapping the spectrum into another with mean level density equal to 1 . The actual energy levels $E_{i}$ are mapped into new dimensionless levels $\epsilon_{i}=\bar{M}\left(E_{i}\right), i=1, \ldots, M$. Here $M$ stands for the dimensionality of the level sequence selected for the analysis, and $\bar{M}(E)=\int_{-\infty}^{E} d E^{\prime} \bar{g}\left(E^{\prime}\right)$ is the smooth part of the accumulated level density $M(E)$, which gives the number of levels up to energy $E$.

The principal difficulty of the unfolding procedure is the correct characterization of the mean level density function $\bar{g}(E)$. Several expressions describing the mean level density of MB systems can be found in the literature [20]. In these expressions, the residual interactions among particles are very often ignored, and the existence of an evenly spaced SP spectrum is assumed, so the formal problem is essentially combinatorial. Recently, a combination of statistical physics methods and the semiclassical approximation has been proposed to take into account the contribution of SP level fluctuations [21]. For fermion systems we can cite the well known formula by Bethe [22]

$$
g(E)=\frac{1}{E \sqrt{48}} \exp \left(\sqrt{2 \pi^{2} \rho_{F} E / 3}\right),
$$

where $\rho_{F}$ is the average SP level density at the Fermi level. The Bethe density diverges when $E$ goes to zero, which of course is meaningless, and reflects the fact that as the excitation energy becomes low, the level density becomes too small to apply statistical methods. We can also cite the so called constant temperature formula [23]

$$
g(E)=\frac{1}{T} \exp \left(\frac{E-E_{0}}{T}\right)
$$

which is also obtained by means of very general statistical arguments. It is used to describe the low-energy level density of atomic nuclei determining $E_{0}$ and $T$ in an empirical way to the experimental data.

Since these expressions are more appropriated either for systems with a large number of particles and high excitation energies or for low excitation energies, we shall proceed in a different way that is better suited for small systems and different energy scales. Actually, the MB level density is defined as

$$
g(E)=\sum_{I} \delta\left(E-E_{I}\right)=\sum_{I}\langle I|\delta(E-\hat{\mathcal{H}})| I\rangle,
$$

and, after inserting Eqs. (3) and (4) into this expression, a straightforward calculation gives

$$
g(E)=\sum_{I} C_{I}^{2} \sum_{P} \phi^{t_{p}}\left(\prod_{i_{j} \in I} \delta_{i_{j} i_{p_{j}}}^{c}\right) \delta\left(E-\sum_{i_{j} \in I} e_{i_{j}}\right),
$$

where $\delta_{i, j}^{c}$ is the Kronecker delta and $p_{j}$ is a permutation of $1,2, \ldots, N$. Removing the restriction that the SP indices must be ordered (i.e., $i_{1} \leqslant i_{2} \leqslant \cdots \leqslant i_{N}$ )

$$
g(E)=\frac{1}{N !} \sum_{\left\{i_{j} \in \mathbb{N}\right\}} \sum_{P} \phi^{t_{p}}\left(\prod_{j=1}^{N} \delta_{i_{j} i_{p_{j}}}^{c}\right) \delta\left(E-\sum_{j=1}^{N} e_{i_{j}}\right) .
$$


In order to express (11) in terms of $\rho(E)$, we use the following property:

$$
\sum_{i, j} \delta\left(E-e_{i}-e_{j}\right)=\rho(E) * \rho(E),
$$

where * stands for the convolution product. Moreover, by decomposing each permutation into $a_{1}, a_{2}, \ldots, a_{N}$ cycles of length $1,2, \ldots, N[24]$, we obtain

$$
g(E)=\sum_{\substack{\left\{a_{r}\right\} \\ \sum r a_{r}=N}}\left(\prod_{r=1}^{N} \frac{\phi^{(r+1) a_{r}}}{r^{2 a_{r} a_{r} !}}\right)\left(* \prod_{r=1}^{N} \prod_{s=1}^{a_{r}} \rho(E / r)\right),
$$

and therefore all the information concerning the level density of the $N$-body system is encoded in the SP level density $g_{S P}(E)=\rho(E)$. In particular, for $N=2$ we get

$$
g(E)=\frac{1}{2} \rho(E) * \rho(E)+\frac{\phi}{4} \rho(E / 2),
$$

an expression that coincides with that quoted in Ref. [8], but within a different context.

Since we assume that the statistical properties of the single-particle spectrum are given by an appropriate matrix ensemble, we expect that a statistical average over the ensemble gives the smooth part of the level density, i.e.,

$$
\bar{g}(E)=\langle g(E)\rangle=\sum_{\substack{\left\{a_{r}\right\} \\ \sum r a_{r}=N}}\left(\prod_{r=1}^{N} \frac{\phi^{(r+1) a_{r}}}{r^{2 a_{r} a_{r} !}}\right)\left\langle * \prod_{r=1}^{N} \prod_{s=1}^{a_{r}} \rho(E / r)\right\rangle,
$$

where $\langle\cdot\rangle$ stands for the statistical average over the ensemble. This equation involves multidimensional integrals of $(1,2, \ldots, N)$-point correlation functions which become extremely complex as $N$ increases. Only Poisson-like fluctuations lead to very simple $k$-point correlation functions for any value of $k$.

Therefore, the need for a useful simplification is clearly seen. Writing the ensemble averages $\left\langle\Pi_{q} \rho\left(x_{q}\right)\right\rangle$ in terms of the $k$-point correlation functions $R_{k}\left(x_{1}, \ldots, x_{k}\right)$, and taking into account that these are positive definite functions, i.e., $0 \leqslant R_{k}\left(x_{1}, \ldots, x_{k}\right) \leqslant \mathcal{M}_{k}, \forall x_{i}$, a cumbersome but straightforward calculation makes it possible to obtain a common upper bound for fermions as well as for boson systems. When $E$ is large enough

$$
\bar{g}(E) \leqslant \frac{1}{N !} \sum_{p=1}^{N}(-1)^{N-p} S_{N}^{(p)}\left(\sum_{k=1}^{p} \mathcal{M}_{k} \mathcal{S}_{p}^{(k)}\right) \frac{E^{p-1}}{(p-1) !},
$$

where $S_{N}^{(p)}$ and $\mathcal{S}_{p}^{(k)}$ are the Stirling numbers of first and second kind, respectively [24]. This upper bound shows that the MB level density can be bounded by a polynomial of degree $N-1$. Moreover, for very small values of $N$ it is possible to obtain exact expressions of the level density. For instance, if $N=2$ and the SP spectrum has GOE spectral fluctuations, we get

$$
\begin{aligned}
\bar{g}(E)= & \frac{E}{2}+\frac{\phi}{4} \\
& +\frac{1+\operatorname{si}(\pi E) \sin (\pi E)-\cos (2 \pi E)-2 \pi E \operatorname{si}(2 \pi E)}{2 \pi^{2} E},
\end{aligned}
$$

where $\operatorname{si}(x)$ is the sine integral function [24].

The dominant part of Eq. (17), a polynomial of degree 1 ( $N-1$ in the general case of $N$ particles), comes directly from the SP smooth level density $\bar{\rho}(E)$. On the other hand, the contribution of $\tilde{\rho}(E)$ to the $\mathrm{MB}$ level density varies as $O(1 / E)$, and thus becomes negligible when $E \gg 1$. A similar result is obtained when $N=3$.

For these reasons we expect that the smooth MB level density $\bar{g}(E)$ can be approximated by a polynomial of degree $k-1$ with $k \simeq N$. Then, the smooth accumulated density $\bar{M}(E)$ will be given by a polynomial of degree $k \simeq N$. Therefore the unfolding procedure can be performed introducing the mean cumulated level density

$$
\bar{M}(k, \alpha, E)=\alpha_{k} E^{k}+\alpha_{k-1} E^{k-1}+\cdots+\alpha_{1} E+\alpha_{0},
$$

and the optimal values $\alpha_{0}$ are obtained by minimizing the distance

$$
\chi(k, \alpha)^{2}=\frac{1}{M} \sum_{i=1}^{M}\left[M\left(E_{i}\right)-\bar{M}\left(k, \alpha, E_{i}\right)\right]^{2} .
$$

In order to see whether the proposed mean cumulated density $\bar{M}(k, \alpha, E)$ is suitable for our problem, we have first performed the fit inside a fixed energy window with $\Delta \epsilon$ $\simeq 100$, common to all the spectra. Since $\bar{M}(k, \alpha, E)$ depends dramatically on $k$ and $E$, this is necessary to compare the fits for systems with different number of particles. Of course the number of levels lying inside this window increases very quickly with $N$ : it varies from $M \simeq 3000$ levels when $N=2$ up to $M \simeq 4 \times 10^{6}$ levels for $N=6$. In Fig. 1 the behavior of $\chi\left(k, \alpha_{0}\right)$ as a function of the degree $k$ in Eq. (18) is shown for systems with different number of fermions moving in a SP spectrum with GOE spectral fluctuations. In all the cases $\chi\left(k, \alpha_{0}\right)$ decreases as $k$ increases until a plateau is reached at $k=N$; for $k \geqslant N$ the value of $\chi\left(k, \alpha_{0}\right)$ remains essentially constant. Moreover, the minimum increases proportionally to $\sqrt{M-(N+1)}$ (shown in the upper right panel), as expected from statistical considerations [25]. This result supports our conjecture that $N$ is the optimal degree of the polynomial (18) and thus we have proceed to fit Eq. (18) to all the sequences considered in the present work.

Figure 2 displays the comparison of a polynomial density, a phenomenological Bethe formula $g(E)=(a / E) \exp (\sqrt{b E})$, and the constant temperature density (8) with the actual density of three fermions evolving in a SP spectrum with GOE spectral fluctuations. The low-energy part of the spectrum has been truncated. We plot the level density functions $g(E)$ instead of $M(E)$ because the latter grows very fast with the energy, and it is quite difficult to distinguish its fluctuations around the mean cumulated level density. It can be seen that 


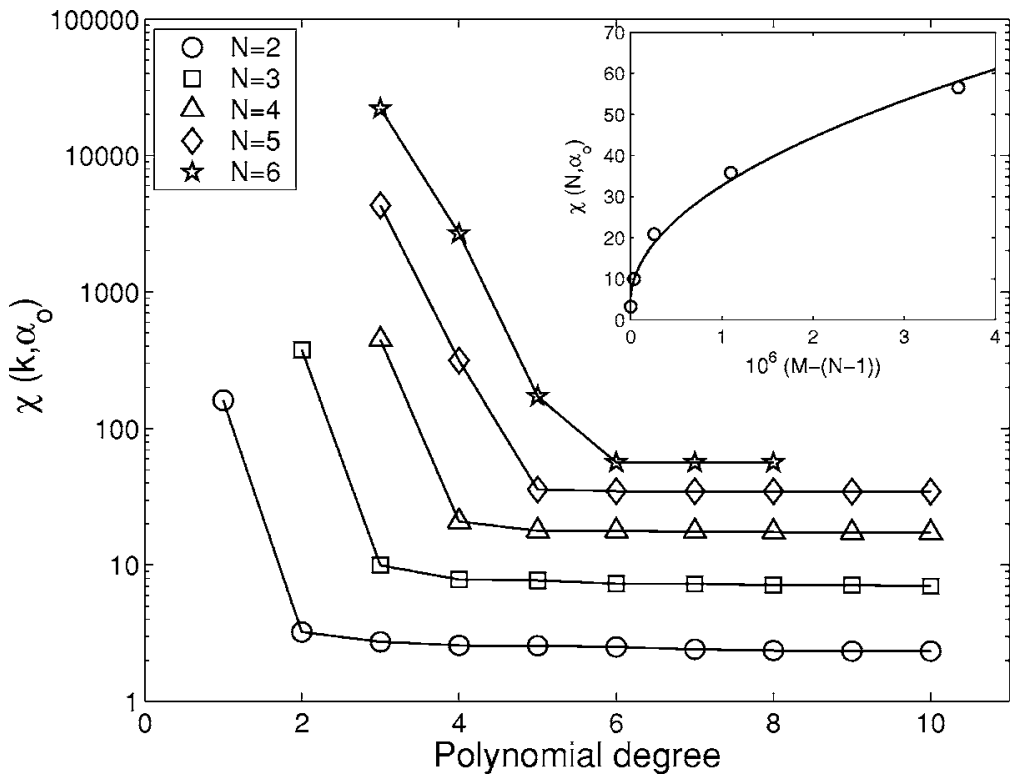

FIG. 1. Minimum distance $\chi\left(k, \alpha_{0}\right)$ between the cumulated density and the approximation (18) to the mean cumulated density, as a function of $k$, the degree of the polynomial, and $N$. The upper right panel shows $\chi\left(N, \alpha_{0}\right)$ versus $M-(N+1)$.
$\bar{g}\left(N, \alpha_{0}, E\right)$ fits very well the actual level density while the other forms give incorrect values almost through the whole energy window. At the spectrum edges they overestimate the average density while in the middle region they give smaller values than the polynomial density. Our results for systems with a larger number of particles show that these discrepancies become smaller as $N$ increases, as expected.

\section{B. The statistical tools}

In order to study the spectral fluctuations of the unfolded spectrum we introduce three convenient statistics.

(i) The nearest neighbor spacing distribution $P(s)$, well suited to study short-range spectral correlations [11], gives the probability that the distance between two consecutive levels lies between $s$ and $s+d s$. For systems with Poisson statistics $P(s)=\exp (-s)$, whereas for chaotic ones it is given by the Wigner distributions $P(s) \propto s^{\nu} \exp \left(-\gamma_{\nu} s^{2}\right)$, with $\nu$ $=1,2$, or 4 , depending on the symmetries of the Hamiltonian. The exponent $\nu$ measures the intensity of the level repulsion characteristic of chaotic systems.

(ii) The $\Delta_{3}$ statistic [26], used to investigate long-range correlations. This function, averaged over intervals, measures the deviations of the unfolded spectrum from a truly equidistant spectrum, i.e., the spectral rigidity. For Wignerlike spectra, the levels are strongly correlated and $\left\langle\Delta_{3}(L)\right\rangle$ $\propto \log L$. By contrast, for Poisson spectra the levels are uncorrelated, the spectrum is soft, and $\left\langle\Delta_{3}(L)\right\rangle \propto L$.

(iii) The power spectrum of the $\delta_{n}$ statistic [27], considered as a discrete time series. It has been recently shown that the power spectrum $P_{k}^{\delta}$ of this statistic exhibits neat $1 / f^{\alpha}$

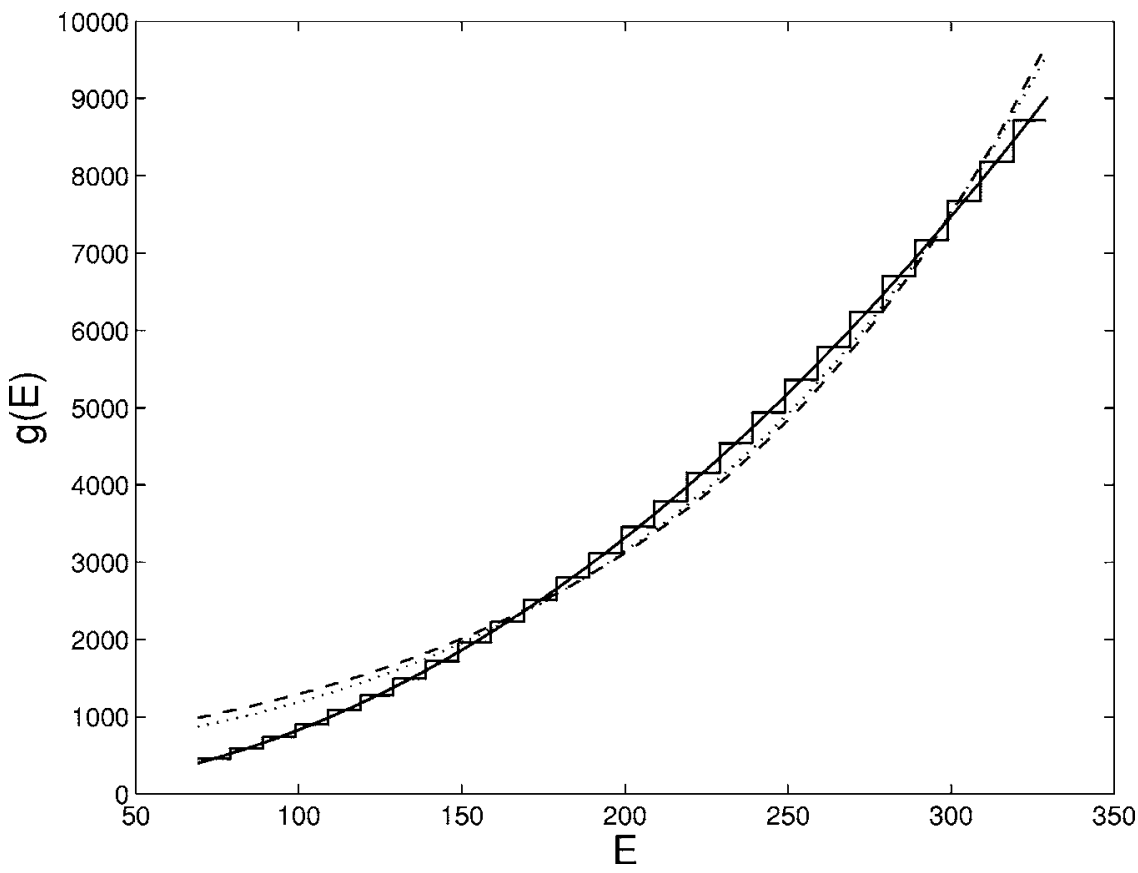

FIG. 2. Level density function (histogram) for a system with $N$ $=3$ fermions in a SP spectrum with GOE fluctuations, together with the best fits to $\bar{g}(N, \alpha, E)$ (solid line), to the phenomenological Bethe density (dotted line), and to the constant temperature formula (dashed line). 


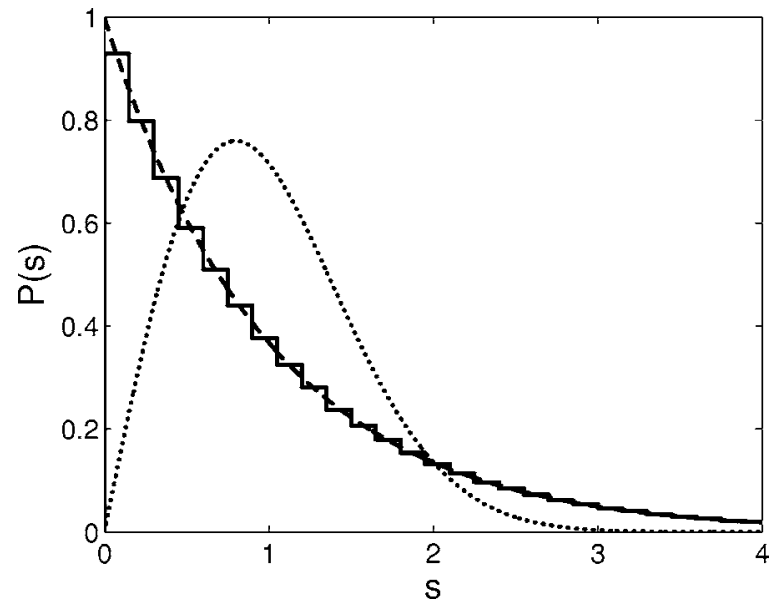

FIG. 3. $P(s)$ distribution for a noninteracting two-fermion system with single-particle spectrum with GOE fluctuations (histogram) compared with the theoretical predictions for Poisson statistics (dashed line) and GOE statistics (dotted line).

power laws, with $\alpha=1$ for chaotic systems and $\alpha=2$ for regular ones [27].

\section{NUMERICAL RESULTS FOR NON-INTERACTING FERMION SYSTEMS}

The following sections are essentially devoted to gain some insight into the spectral fluctuation properties of NIP systems by means of numerical simulations. We consider separately the simulations for fermion and boson systems. Here we study the spectral fluctuations of small systems composed by a few noninteracting and spinless fermions, while boson systems are studied in the next section. The relevant difference between these systems is that the Pauli principle is active only for fermions, and therefore the MF levels are occupied at most by a single particle.

\section{A. Evolution with the number $N$ of particles}

We first deal with the statistical properties of the whole spectrum. In order to obtain significant results we generate very long and complete sequences of energy levels corresponding to many-body systems with $N=2, \ldots, 6$ fermions. Since we expect to obtain different results in the low energy region, we postpone its study until the next subsection. Using the procedure explained in Sec. II, we generate sequences of about one million levels, and afterwards several thousands of the lower levels are removed to keep exactly one million. Moreover, in doing so, we can take advantage of the approximated smooth level density (18) to unfold the truncated spectrum.

Since the results for different symmetries and different numbers of particles are very similar, we only show a few representative examples.

\section{Short-range correlations}

The $P(s)$ distribution of two systems with $N=2$ and 3 particles in a SP spectrum with GOE spectral fluctuations are

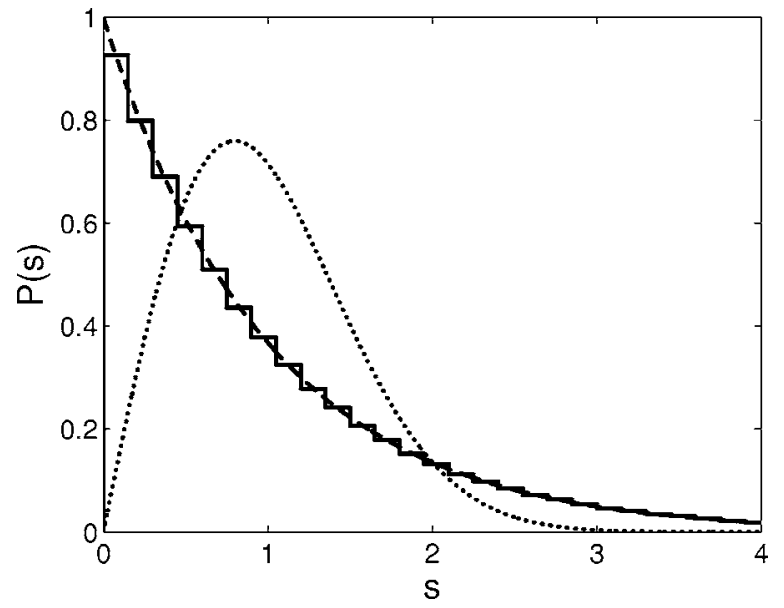

FIG. 4. Same as Fig. 3 for $N=3$ particles.

compared in Figs. 3 and 4 with the Poisson and Wigner distributions. It appears that both systems show short-range spectral fluctuations characteristic of regular systems.

To be more quantitative we can fit the $P(s)$ histograms to the Brody distribution $P(\nu, s)[11]$, where $\nu$ is the so-called Brody parameter. The very interesting feature of this distribution is that it interpolates between the Poisson distribution $(\nu=0)$ of regular systems and the Wigner distribution $(\nu$ $=1$ ), which is a good approximation to the GOE spacing distribution. Thus, the degree of chaos can be assessed by how close $\nu$ is to these limits. Table I displays the values of the Brody parameter $\nu$ for several few-body systems, and in all the cases it is close to 0 with very high precision. This result proves that the spectrum becomes completely regular when we add just one particle to the SP system, at least according to the short-range spectral correlations.

\section{Long-range correlations}

In order to confirm this result, we try to study the longrange correlation structure of the spectrum. It is quite customary to use the average values of the $\Delta_{3}(L)$ statistic. After generating the spectra of NIF systems, where the SP spectrum exhibits GUE fluctuations, the numerical values of $\left\langle\Delta_{3}(L)\right\rangle$ were calculated as averages over semioverlapping intervals of length $L$ covering the whole level sequence [3]. Figure 5 compares the numerical results for $N=2$ and 3 with the theoretical predictions for integrable systems up to $L$ $=200$. We see that in both cases $\left\langle\Delta_{3}(L)\right\rangle$ increases linearly in perfect agreement with the theoretical predictions for inte-

TABLE I. Brody parameter values for several noninteracting fermion systems with $N=1$ to 6 particles and SP spectrum with GOE fluctuations. $\Delta \nu$ is an estimate of the statistical error committed in the fit.

\begin{tabular}{ccccccc}
\hline \hline$N$ & 1 & 2 & 3 & 4 & 5 & 6 \\
\hline$\nu$ & 0.961 & 0.0000 & 0.000 & 0.00000 & 0.000 & 0.0000 \\
$\Delta \nu$ & 0.009 & 0.0003 & 0.004 & 0.00007 & 0.004 & 0.0001 \\
\hline \hline
\end{tabular}



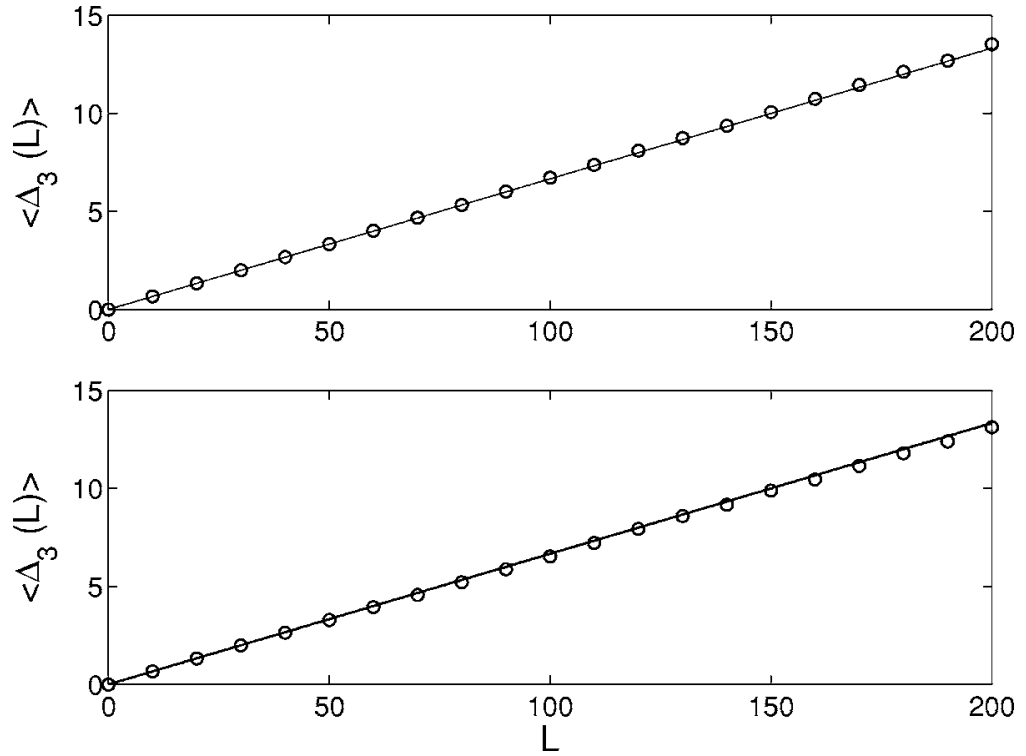

FIG. 5. Average $\Delta_{3}(L)$ values for noninteracting few-fermion systems with $N=2$ and 3 particles generated from a single-particle spectrum with GUE fluctuations (circles). The numerical values are compared with the Poisson limit $L / 15$ (solid line). The GUE curve is not shown because it is nearly two orders of magnitude smaller than the calculated values. grable systems. Beyond $L \simeq 200$ it is quite difficult to obtain reliable results for $\left\langle\Delta_{3}(L)\right\rangle[28,29]$.

A second way to characterize long-range correlations is to use the average values $\left\langle P_{k}^{\delta}\right\rangle$ of the power spectrum of $\delta_{n}$. In this case, the independent variable is the counter $k$ related to the actual frequency as $\omega_{k}=2 \pi k / M$, where $M$ is the total length of the level sequence. The frequency counter takes on the values $k=1,2, \ldots, M / 2$. The $\left\langle P_{k}^{\delta}\right\rangle$ values are obtained by means of a double average. First, an "ensemble" average is obtained using 100 intervals of length $L=10^{4}$ that cover the whole level sequence; then the logarithmic frequency axis is divided into equal bins and the power spectrum components are averaged in each bin. Figure 6 compares $\left\langle P_{k}^{\delta}\right\rangle$ for the same previous systems with $N=2$ and $N=3$ with the theoretical predictions given in Ref. [31]. Again, the agreement with the prediction for Poisson statistics is almost perfect. However, we have found that the average power spectrum bends down below a very small critical frequency $k_{c}$. There exists a one-to-one correspondence between the frequency $k$ and a characteristic energy scale in the spectrum $L$, given by $L$ $=M / k$; then small frequencies in the $\delta_{n}$ power spectrum correspond to large values of $L$. The $k_{c}$ values must be estimated before the bin average of the power spectrum. When $N=2$, the calculated points deviate from theoretical line below $k_{c}$ $\simeq 12$, corresponding to a critical energy window $L_{c} \simeq 800$. Analogously, if $N=3$ the departure from the theoretical line is only seen for frequencies below $k_{c} \simeq 3$, and thus $L_{c}$ $\simeq 3000$. Finally, for $N>3$ the critical length $L_{c}>M$ and therefore the results are indistinguishable from the theoretical curve. One important point is that the low-frequency deviations from the theoretical predictions appear only when the SP spectrum exhibits RMT-like fluctuations. If this statistic is calculated for systems with an integrable MF, the agreement with the theoretical predictions is excellent, even at very low frequencies. Therefore, these discrepancies are most likely a reminiscence of the Wigner-Dyson spectral fluctuations of the SP spectrum. These results show that NIF
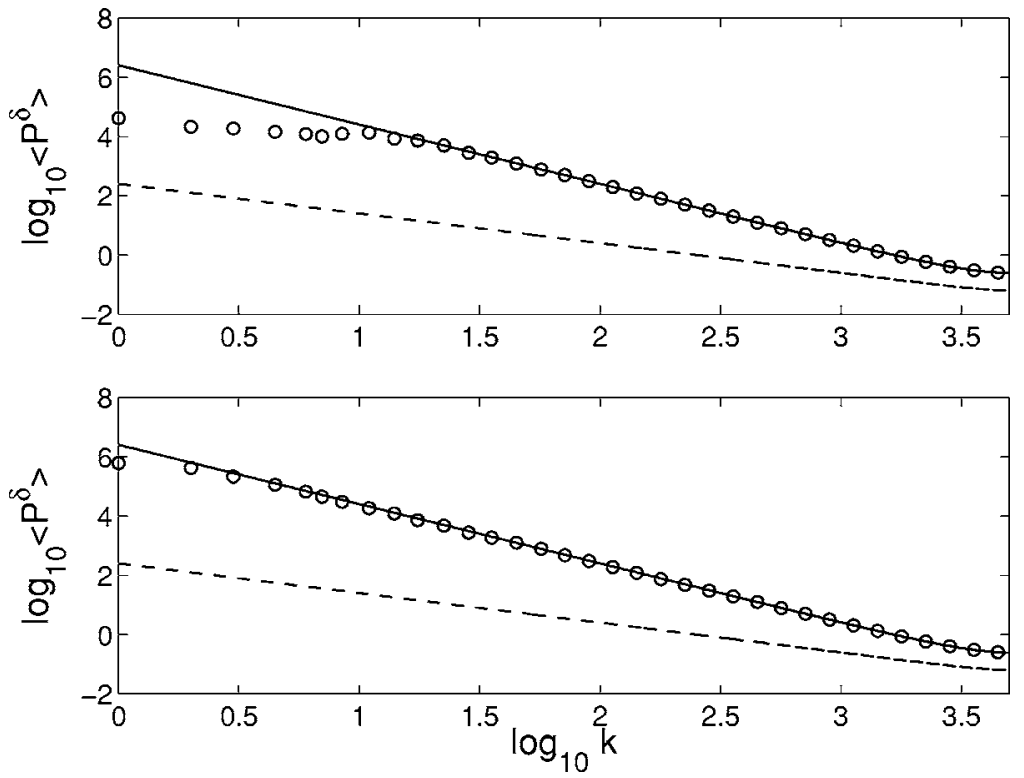

FIG. 6. Average $P_{k}^{\delta}$ values for noninteracting $N=2$ and 3 fermion systems generated from a single-particle spectrum with GUE fluctuations (circles), compared with the theoretical predictions for GUE (dashed line), and regular systems (solid line). 
systems exhibit long-range correlation characteristic of integrable systems. Nevertheless, for chaotic MF this assessment is only true up to a certain critical energy window $L_{c}$. A more detailed analysis of the dependence of $L_{c}$ on $N$ and $E$ is left for the next subsection.

\section{B. Evolution with the energy}

The statistical properties of the low-energy region were not considered in the previous analysis. As was argued in the Introduction, we expect a different behavior in this case since Bloch's argument may fail for the low-lying levels of the MB spectrum. It is not clear whether the SP levels defining two near and low-lying many-body configurations come from different regions of the SP spectrum, so they can not be considered as uncorrelated. Indeed, we can find a different behavior for the very low-lying levels as it is shown in the following example. Given a SP spectrum with GOE-like fluctuations we consider the ground-state and the first excited energy levels of the two-body spectrum. The ground-state configuration consist of one particle occupying the first SP level and the other the second SP level. Analogously, the first and the third SP levels are occupied in the first excited configuration. Then, the energy spacing between these two consecutive levels of the two-body spectrum $S_{1}$ is equal to the second energy spacing $s_{2}$ of the SP spectrum. Thus, if we calculate the nearest neighbor spacing distribution by averaging $S_{1}$ over different SP spectra we would obtain a Wigner distribution.

Before going on with the statistical analysis, it is worth commenting briefly on the unfolding procedure of the low energy spectrum. As it was already pointed out, the polynomial approximation (18) is appropriated for higher energies. For this reason we have calculated the smooth cumulated density $\bar{M}(E)$ by means of an ensemble average. This was obtained using as many as 500 SP spectra.

As usual, we start considering the short-range correlations. To this purpose we have studied the evolution of the $P(s)$ distribution with the threshold energy $E_{\max }$ for a system with $N=2$ fermions moving freely in a GOE MF. The shape of $P(s)$ is compared in Fig. 7 with the theoretical predictions for integrable and chaotic spectra. The figure is separated into four different panels according to the values of $E_{\max }$. It is clear that $P(s)$ evolves smoothly from the Wigner distribution to the Poisson distribution as the energy increases. While at very low energies $\left(E_{\max }=4\right) P(s)$ does coincide with the former, it shows an intermediate behavior when we move up to $E_{\max }=10$, and only near $E_{\max }=40$ becomes almost indistinguishable from the Poisson distribution. Even in this case, there exist small deviations that disappear when the threshold energy increases up to $E_{\max }=160$. At this point we can state that as far as short-range correlations are concerned, Bloch's argument fails at low energies.

It is also worthwhile to comment on the actual shape of this intermediate distributions for the $P(s)$. The value of the $P(s)$ when $s=0$ is different from 0 in the distributions going from Wigner to Poisson as can be clearly seen in the second panel of Fig. 7. This kind of behavior appears when part of the neighboring levels are correlated and present normal
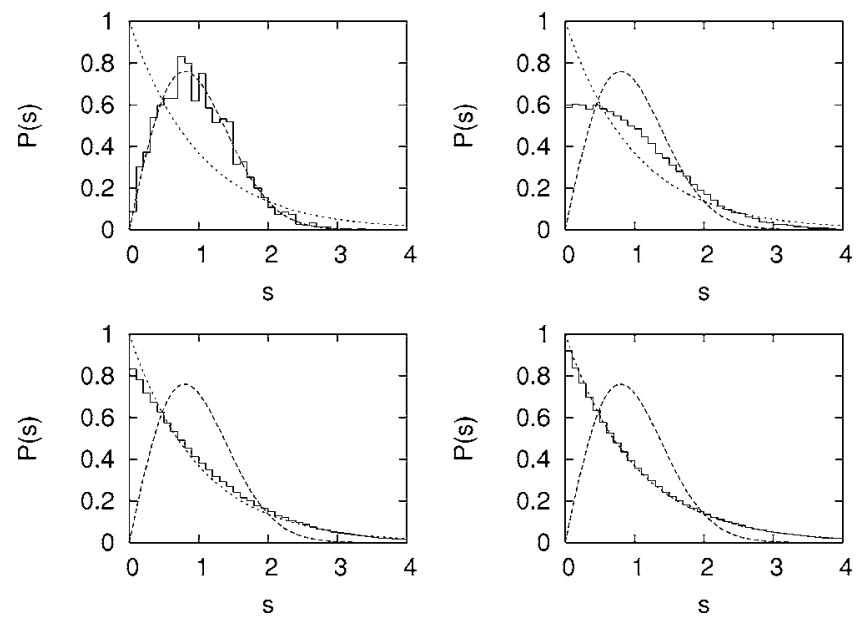

FIG. 7. Evolution of the $P(s)$ distribution with the energy threshold $E_{\text {max }}$ for a two-body system. Using four different energies $E_{\text {max }}=4$ (upper left panel), 10 (upper right panel), 40 (lower left panel), and 160 (lower right panel), the actual distribution (histogram) is compared with the Wigner (dashed line) and with the Poisson distribution (dotted line).

level repulsion, and part of them do not repel each other and follow Poisson statistics. It is very similar to the behavior in mixed systems when part of the phase space is chaotic and part is regular and they are isolated from each other [30]. On the contrary, it is very different from the usual behavior appearing in many-body systems where the probability of finding two consecutive levels degenerated is zero, when we turn on the interaction smoothly.

In order to complete the study of the evolution of the spectral fluctuations, we shall characterize the behavior of $\left\langle P_{k}^{\delta}\right\rangle$ at different excitation energies in the MB spectrum. When the low energy domain is excluded, the average power spectrum of $\delta_{n}$ is in perfect agreement with the prediction for Poisson statistics except at small frequencies. As we have seen in Fig. 6 this frequency is typically $k_{c} \in[1,10]$ for systems with $N=2,3$ particles. Thus, the spectral fluctuations of these systems deviate from those of integrable systems for energy windows of length $L_{c} \in[1000,10000]$. As for the shape of $P(s)$, we expect the critical value $L_{c}$ to depend on the energy. To get an idea of how $L_{c}$ varies with the energy, we have computed the power spectrum of $\delta_{n}$ for different energy intervals $[E-E / 10, E+E / 10]$ and several numbers of particles. The evolution can be followed in the three panels of Fig. 8 corresponding to $E=1000,8000$, and 64000 . In each panel we compare the results for $N=2,3$, and 4 with the theoretical curve for integrable systems; the latter as well as the numerical results are displaced in the vertical axes to avoid overlapping. There are two features that the reader has probably noticed already. The sequences of $\left\langle P_{k}^{\delta}\right\rangle$ values are longer as the energy increases because the number of energy levels involved increases with $E$ very quickly. On one side, the length of the energy interval we use to calculate the power spectrum is proportional to $E$, and on the other the level density also increases with the energy. There is also a difference between Figs. 8 and 6. In order to make clearer the dependence of $L_{c}$ with the energy window we have plot- 

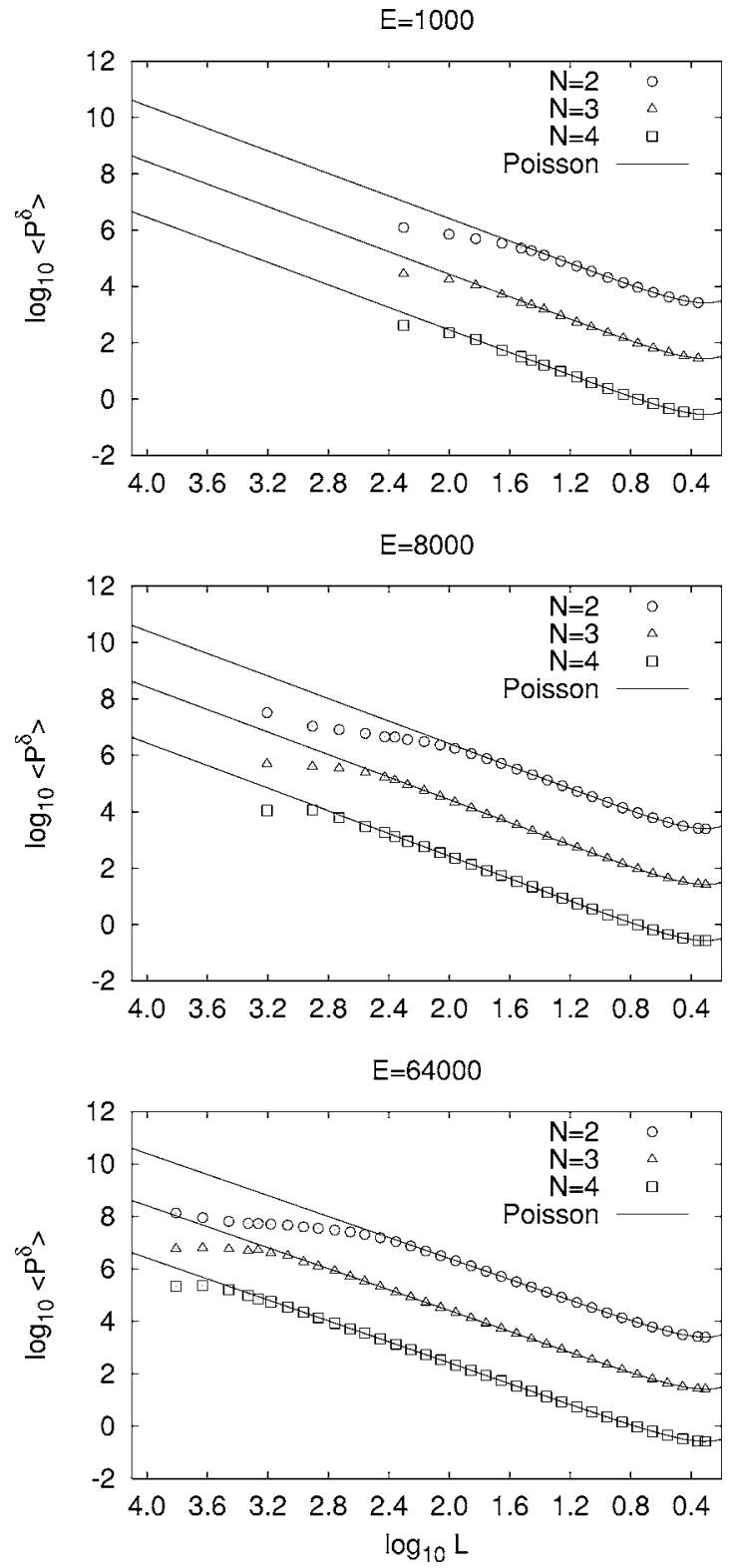

FIG. 8. $\left\langle P_{k}^{\delta}\right\rangle$ behavior for noninteracting $N=2$ (circles), 3 (triangles), and 4 (squares) fermion systems at different energies, compared with the theoretical predictions for regular systems (solid line). At each energy, the numerical values and the theoretical line are appropriately displaced to avoid overlapping.

ted $\left\langle P_{k}^{\delta}\right\rangle$ as a function of $L=M / k$. It is clearly seen that for a given energy $E, L_{c}$ increases with the number of particles; and for a fixed number of particles $N, L_{c}$ increases with energy. Table II gives the $L_{c}$ estimates for $N=2$ and 4 .

Unlike the behavior of $P(s)$ at very low energies, the deviation of $\left\langle P_{k}^{\delta}\right\rangle$ from the Poisson statistics prediction for $L$ $>L_{c}$ is not a signature of an intermediate regime. For a recent study of the behavior of the $\delta_{n}$ in the order to chaos transition see Refs. [32,33]. On the contrary, this sharp change of slope of the power spectrum seems to be a reminiscence of the GOE-like fluctuations of the SP spectrum.
TABLE II. Approximated $L_{c}$ values for systems with $N=2$ and 4 particles as a function of the energy window centroid $E$.

\begin{tabular}{ccc}
\hline \hline & \multicolumn{2}{c}{$L_{c}$} \\
\cline { 2 - 3 }$E$ & $N=2$ & $N=4$ \\
\hline 1000 & 60 & 200 \\
8000 & 130 & 1200 \\
64000 & 400 & 5000 \\
\hline \hline
\end{tabular}

\section{NUMERICAL RESULTS FOR NONINTERACTING BOSON SYSTEMS}

The analysis of the preceding section is repeated for systems of $N$ bosons without interaction. Since the exclusion principle does not apply to boson systems, the sequence $I$ $=\left\{i_{1}, i_{2}, \ldots, i_{N}\right\}$ may contain sets of equal indices, i.e., SP levels can have occupancies greater than one; actually one level may be occupied by $N$ particles. This property can lead to a different behavior of spectral fluctuations when $N$ increases and $m$ remains constant. Nevertheless, for infinite systems, where $m \rightarrow \infty$ for $N$ fixed we do not expect many differences with regard to fermion systems. To be concise we only report the results for $N=2$; the spectral fluctuations of systems with $N=3,4,5$ and 6 particles show a very similar behavior.

The number $m$ of SP levels is taken as to generate complete sequences slightly larger than one million levels; then we truncate the low-energy spectrum to obtain sequences with one million levels exactly. The unfolding of these sequences is performed by using the polynomial density (18).

The $P(s)$ distribution of a system with $N=2$ identical bosons moving freely in a SP spectrum with GOE spectral fluctuations is compared in Fig. 9 with the Poisson and Wigner distributions. It is clearly seen that the short-range spectral fluctuations of this system are those of a regular system. A least-squares fit of the $P(s)$ histograms to the Brody distribution $P(\nu, s)$ [11], gives a repulsion parameter $\nu=0.0000 \pm 0.0003$. Similarly to the case of fermion systems, adding just a particle to the SP system the spectrum becomes completely regular. The $P(s)$ distribution for systems with $N=3,4,5$, and 6 bosons also follow very precisely the Poisson curve.

The study of the long-range correlation structure of the spectrum corroborates the integrable character of the $N=2$ system. According to Fig. 10 the average values $\left\langle\Delta_{3}(L)\right\rangle$ (calculated by using the algorithm of Ref. [3]) increase almost linearly in agreement with the theoretical predictions for integrable systems. Only near the upper limit of the figure $(L$ $=200$ ), the calculated values seem to fall slightly below the theoretical line suggesting the onset of a $\Delta_{3}(L)$ saturation. The existence of such a saturation is studied by means of the $\delta_{n}$ statistic. The whole spectrum is divided in 100 nonoverlapping sequences of length $M=10^{4}$; then the power spectrum of the $\delta_{n}$ statistic is calculated for each sequence separately, and an "ensemble" average is performed by using the 100 sequences. In order to reduce even more the fluctuations 


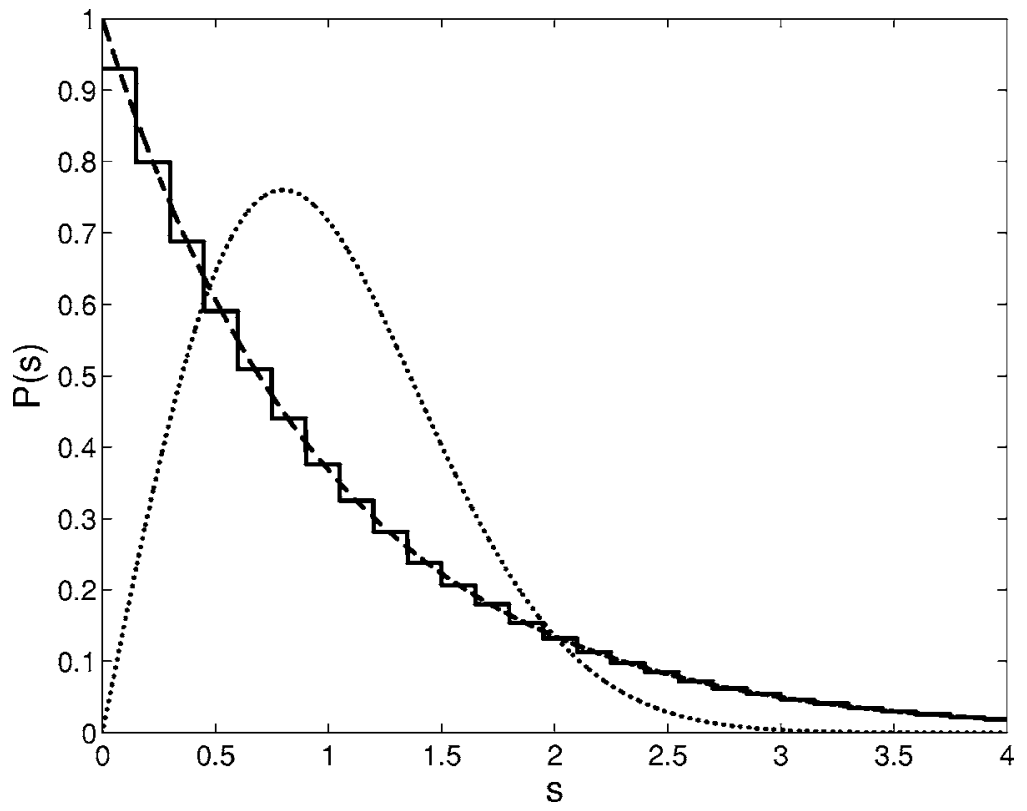

FIG. 9. $P(s)$ distribution for a two-boson system without interaction and a single-particle spectrum with GOE fluctuations (histogram) compared with the theoretical predictions for Poisson statistics (dashed line) and GOE statistics (dotted line).

and clarify the main trend we divide the logarithmic frequency axis into equal bins and average the power spectrum components in each bin. Figure 11 shows that $\left\langle P_{k}^{\delta}\right\rangle$ behaves consistently. It is clearly observed that there exists a critical frequency $k_{c} \simeq 17$; for $k \geqslant k_{c}$ the power spectrum behaves as $\left\langle P_{k}^{\delta}\right\rangle \propto 1 / k^{2}$ in agreement with the theoretical predictions given in Ref. [31] for integrable systems, while below $k_{c}$ it behaves as $\left\langle P_{k}^{\delta}\right\rangle \propto 1 / k$, which is the expected behavior of a chaotic system. The frequency $k_{c}$ corresponds to a critical energy window $L_{c}=M / k_{c} \simeq 600$. Similar results are obtained when $N>2$, but the critical frequency decreases very quickly with $N$. As a few examples, we have $k_{c}=3,2$ for $N=3$ and 4, respectively.

We now study the low-energy region of the spectrum that was excluded before. We expect a different behavior in this region since the arguments that were applied to fermion systems are also valid for bosons. To unfold the spectra in this region, where the polynomial density is not valid, we use an ensemble average with 500 different GOE SP spectra.

The evolution of the $P(s)$ distribution with the threshold energy $E_{\max }$ has been studied for a system with $N=2$ bosons in a GOE MF. Figure 12 compares the calculated shape of $P(s)$ with the theoretical curves for integrable and chaotic spectra. The figure, separated in four different panels according to the values of $E_{\max }$, shows that this distribution evolves smoothly from the Wigner to the Poisson distribution as the excitation energy increases. For $E_{\max }=4, P(s)$ does coincide with the Wigner distribution, it shows an intermediate behavior when we move up to $E_{\max }=10$, and only near $E_{\max }=40$ becomes almost indistinguishable from the Poisson distribution. Even for these energies, there exist small deviations that disappear when the threshold energy moves up to $E_{\max }$ $=160$. Similarly to what happens for fermion systems, we

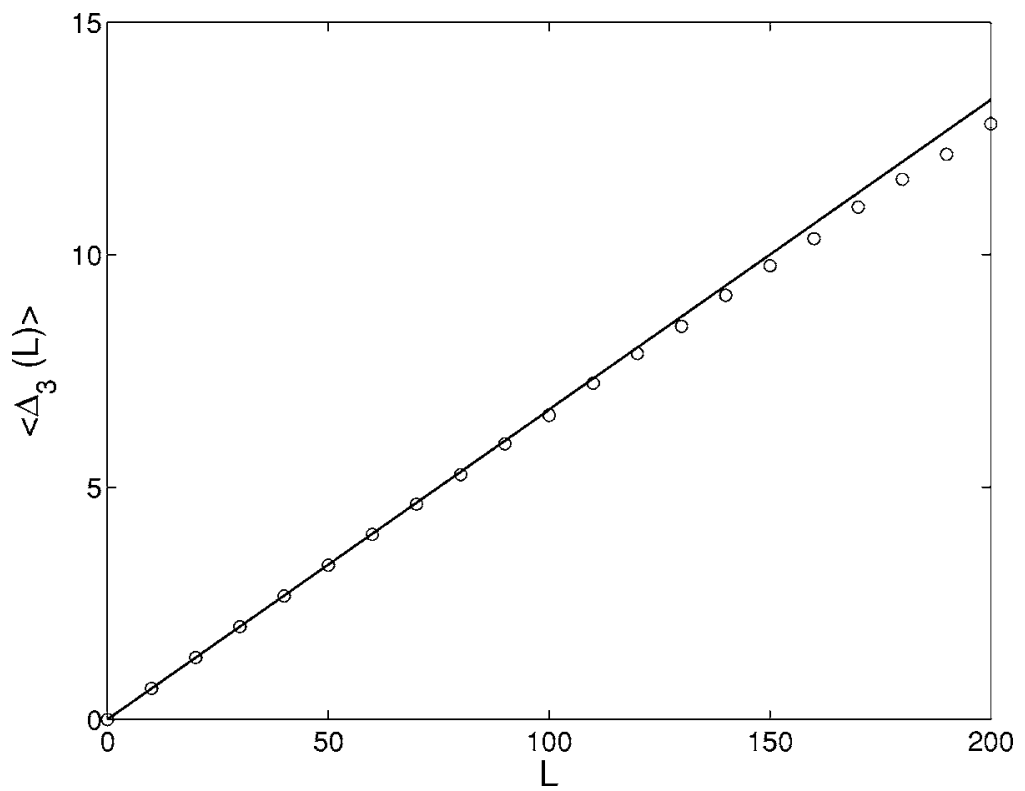

FIG. 10. Average $\Delta_{3}(L)$ values for noninteracting boson systems with $N=2$ particles, generated from a single-particle spectrum with GOE fluctuations (circles). The numerical values are compared with the Poisson limit L/15 (solid line). The GOE curve is not shown because it is nearly two orders of magnitude smaller than the calculated values. 


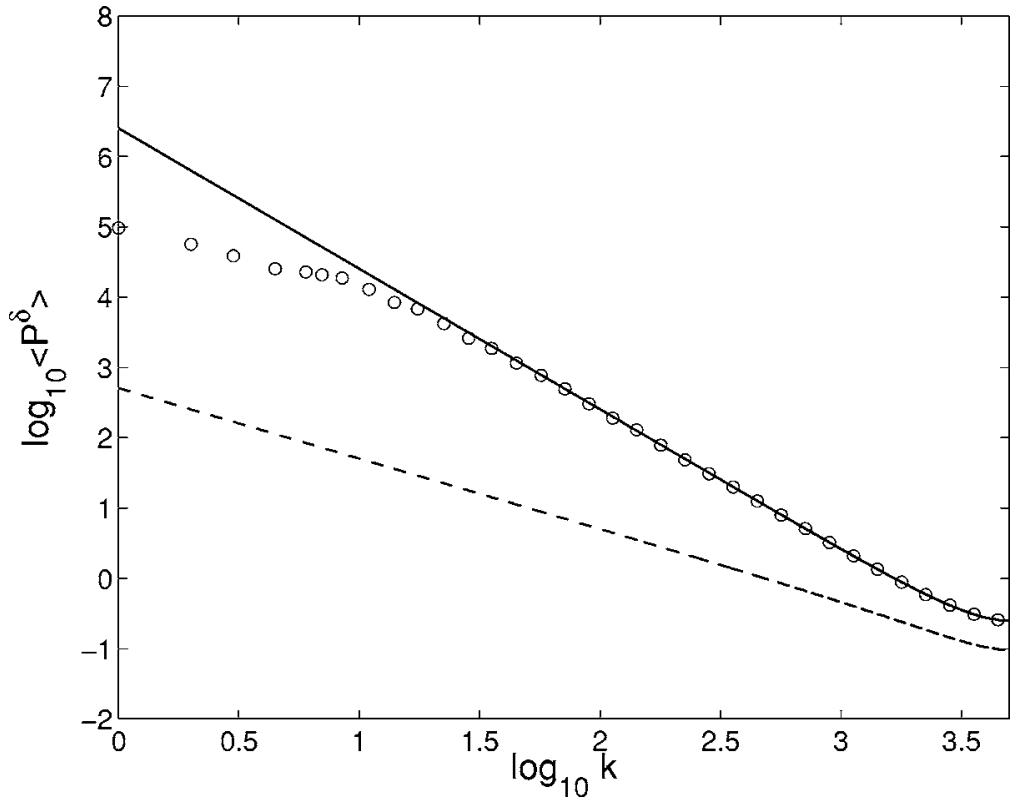

FIG. 11. Average $P_{k}^{\delta}$ values for noninteracting $N=2$ boson systems generated from a singleparticle spectrum with GOE fluctuations (circles), compared with the theoretical predictions for GOE (dashed line), and regular systems (solid line). can state that short-range correlations show that Bloch's argument fails at low energies.

In order to complete the study of the evolution of the spectral fluctuations, we study the behavior of $\left\langle P_{k}^{\delta}\right\rangle$ at different excitation energies. We know already that except at small frequencies the average power spectrum of $\delta_{n}$ is in perfect agreement with the prediction of Poisson statistics, when the low energy domain is excluded. As we have seen in Fig. 6 these frequencies are typically $k \leqslant k_{c} \in[1,10]$ for systems with a small number of particles. Thus, the spectral fluctuations of these systems deviate from those of integrable systems for energy windows of length $L_{c} \in[1000,10000]$. Accordingly to the $P(s)$ results, we expect the critical value $L_{c}$ to depend on the energy. Therefore, we have computed the power spectrum of $\delta_{n}$ for different energy intervals [E
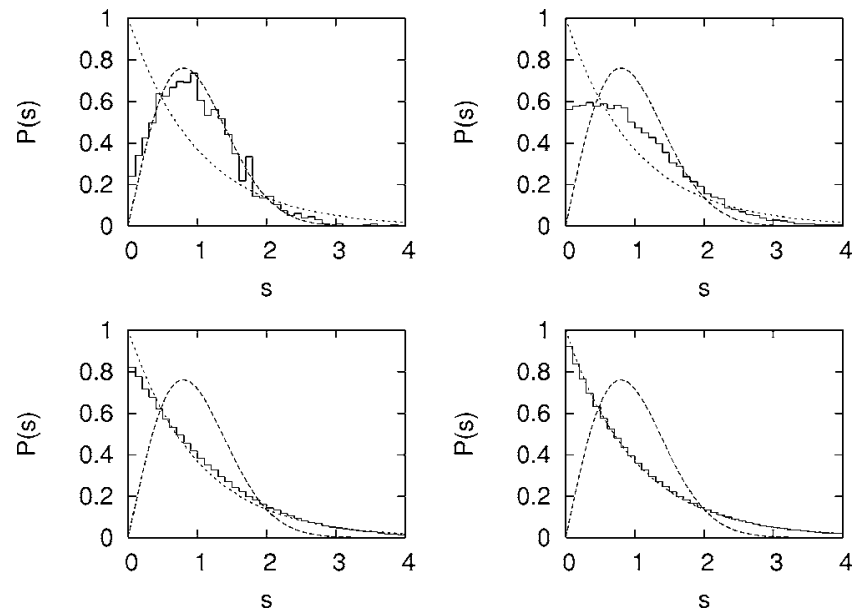

FIG. 12. Evolution of the $P(s)$ distribution with the energy threshold $E_{\max }$ for a two-body system. Using four different energies $E_{\max }=4$ (upper left panel), 10 (upper right panel), 40 (lower left panel), and 160 (lower right panel), the actual distribution (histogram) is compared with the Wigner (dashed line) and with the Poisson distribution (dotted line).
$-E / 10, E+E / 10]$ and several numbers of particles. Figure 8 displays the results for $N=2$ and $E=1000, E=8000$ and $E$ $=64000$. Figure 13 compares the numerical results with the theoretical curve for integrable systems; this one as well as the numerical results are displaced in the vertical axes to avoid overlapping. It is clearly seen that $L_{c}$ increases with energy. The general result is that for a given energy $E, L_{c}$ increases with the number of particles; and for a fixed number of particles $N, L_{c}$ increases with energy. Table III gives the $L_{c}$ estimates for $N=2$ and 4.

\section{AN ANALYTICAL PROOF FOR $N=2$}

In order to obtain some analytical support for this result, we have tried to calculate the two-point cluster functions $Y_{2}^{(N)}\left(E_{1}, E_{2}\right)$ of the system with $N$ particles. However, since this calculation requires the knowledge of the $2 \mathrm{~N}$-point correlation functions for the SP spectrum, it becomes very cumbersome and involved. For this reason we have restricted

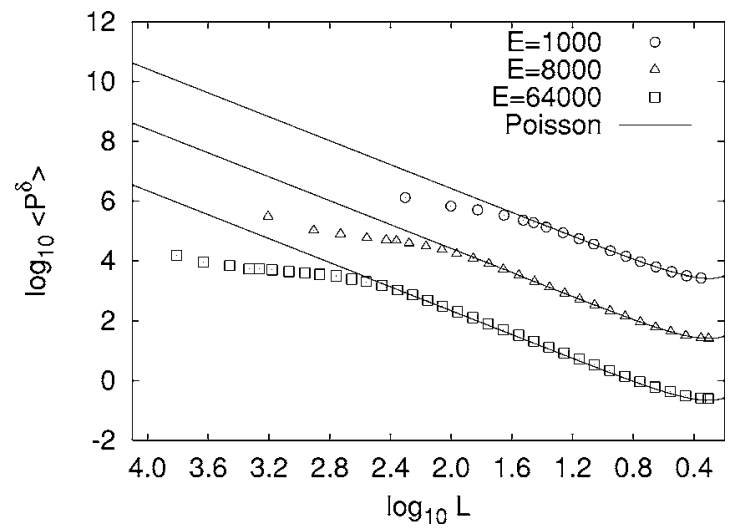

FIG. 13. $\left\langle P_{k}^{\delta}\right\rangle$ behavior for noninteracting $N=2$ boson systems (circles) compared with the theoretical predictions for regular systems (solid line). 
TABLE III. Approximated $L_{c}$ values for boson systems with $N=2$ and 4 particles as a function of the energy window centroid $E$.

\begin{tabular}{ccc}
\hline \hline & \multicolumn{2}{c}{$L_{c}$} \\
\cline { 2 - 3 }$E$ & $N=2$ & $N=4$ \\
\hline 1000 & 40 & 200 \\
8000 & 130 & 800 \\
64000 & 200 & 3200 \\
\hline \hline
\end{tabular}

ourselves to systems with $N=2$ particles. In this case the two-point cluster function is given by

$$
Y_{2}^{(2)}\left(E_{1}, E_{2}\right)=1-\frac{\left\langle g^{(2)}\left(E_{1}\right) g^{(2)}\left(E_{2}\right)\right\rangle}{\left\langle g^{(2)}\left(E_{1}\right)\right\rangle\left\langle g^{(2)}\left(E_{2}\right)\right\rangle}+\frac{\delta\left(E_{1}-E_{2}\right)}{\left\langle g^{(2)}\left(E_{1}\right)\right\rangle},
$$

where $g^{(2)}(E)$ is the two-body level density. In what follows we shall assume that $E_{1}, E_{2} \gg 1$ and $E_{1} \neq E_{2}$. With this proviso the average level density can be approximated by $\left\langle g^{(2)}(E)\right\rangle=E / 2$. We also assume the energies $E_{1}$ and $E_{2}$ to be close, that is, $0<\left|E_{1}-E_{2}\right| \ll E_{1}+E_{2}$, since in the opposite case the energy levels are supposed to be uncorrelated when they are far enough. Inserting Eq. (14) and expressing $\left\langle\rho\left(E_{1}\right) \rho\left(E_{2}\right) \cdots \rho\left(E_{v}\right)\right\rangle$ in terms of $k$-point correlation functions of the SP spectrum $R_{k}\left(E_{1}, E_{2}, \ldots, E_{k}\right)(k \leqslant v)$, we arrive at

$$
\begin{aligned}
Y_{2}^{(2)}\left(E_{1}, E_{2}\right) \\
=1-\frac{1}{E_{1} E_{2}}\left(\int_{0}^{E_{1}} d \eta \int_{0}^{E_{2}} d \lambda R_{4}\left(\eta, E_{1}-\eta, \lambda, E_{2}-\lambda\right)\right. \\
\quad+2 \int_{0}^{E_{1}} d \eta R_{3}\left(\eta, E_{1}-\eta, E_{2}-\eta\right) \\
+2 \int_{0}^{E_{1}} d \eta R_{3}\left(\eta, E_{1}-\eta, \eta-E_{1}+E_{2}\right) \\
\left.\quad+\frac{1}{4} R_{2}\left(E_{2} / 2, E_{1} / 2\right)\right)
\end{aligned}
$$

for fermion systems, and

$$
\begin{aligned}
& Y_{2}^{(2)}\left(E_{1}, E_{2}\right) \\
& =1-\frac{1}{E_{1} E_{2}}\left(\int_{0}^{E_{1}} d \eta \int_{0}^{E_{2}} d \lambda R_{4}\left(\eta, E_{1}-\eta, \lambda, E_{2}-\lambda\right)\right. \\
& \quad+2 \int_{0}^{E_{1}} d \eta R_{3}\left(\eta, E_{1}-\eta, E_{2}-\eta\right) \\
& \quad+2 \int_{0}^{E_{1}} d \eta R_{3}\left(\eta, E_{1}-\eta, \eta-E_{1}+E_{2}\right) \\
& \quad+\int_{0}^{E_{2}} d \eta R_{3}\left(E_{1} / 2, \eta, E_{2}-\eta\right)
\end{aligned}
$$

$$
\begin{aligned}
& +\int_{0}^{E_{1}} d \eta R_{3}\left(E_{2} / 2, \eta, E_{1}-\eta\right) \\
& +2 R_{2}\left(E_{2} / 2, E_{1}-E_{2} / 2\right)+2 R_{2}\left(E_{1} / 2, E_{2}-E_{1} / 2\right) \\
& \left.+\frac{3}{4} R_{2}\left(E_{2} / 2, E_{1} / 2\right)\right)
\end{aligned}
$$

for boson systems. Now, taking into account that the $k$-point correlation functions are bounded, it is possible to drop all except the two first terms in these equations, as far as we are interested in the limit $E_{1}, E_{2} \gg 1$. In this case the remaining terms are of order $O(1 / E)$. Thus

$$
\begin{aligned}
& Y_{2}^{(2)}\left(E_{1}, E_{2}\right) \\
& \quad \sim 1-\frac{1}{E_{1} E_{2}} \int_{0}^{E_{1}} d \eta \int_{0}^{E_{2}} d \lambda R_{4}\left(\eta, E_{1}-\eta, \lambda, E_{2}-\lambda\right) .
\end{aligned}
$$

The integrand $R_{4}\left(E_{1}, E_{2}, E_{3}, E_{4}\right)$ can be written in terms of the level cluster functions $Y_{k}$ with $k \leqslant 4$ as

$$
\begin{aligned}
R_{4}\left(E_{1}, E_{2}, E_{3}, E_{4}\right)= & -Y_{4}\left(E_{1}, E_{2}, E_{3}, E_{4}\right) \\
& +\left[Y_{3}\left(E_{1}, E_{2}, E_{3}\right) Y_{1}\left(E_{4}\right)+3 \text { perms }\right] \\
& -\left[Y_{2}\left(E_{1}, E_{2}\right) Y_{1}\left(E_{3}\right) Y_{1}\left(E_{4}\right)+5 \text { perms }\right] \\
& +Y_{1}\left(E_{1}\right) Y_{1}\left(E_{2}\right) Y_{1}\left(E_{3}\right) Y_{1}\left(E_{4}\right)
\end{aligned}
$$

giving

$$
\begin{aligned}
Y_{2}^{(2)}\left(E_{1}, E_{2}\right) \sim & 1+\frac{1}{E_{1} E_{2}} \int_{0}^{E_{1}} d \eta \int_{0}^{E_{2}} d \lambda\left[Y _ { 4 } \left(\eta, E_{1}-\eta, \lambda, E_{2}\right.\right. \\
& -\lambda)-Y_{3}\left(E_{1}-\eta, \lambda, E_{2}-\lambda\right)-Y_{3}\left(\eta, \lambda, E_{2}-\lambda\right) \\
& -Y_{3}\left(\eta, E_{1}-\eta, E_{2}-\lambda\right)-Y_{3}\left(\eta, E_{1}-\eta, \lambda\right) \\
& -Y_{2}\left(2 \eta-E_{1}\right) Y_{2}\left(2 \lambda-E_{2}\right)-Y_{2}(\eta-\lambda) Y_{2}\left(E_{1}\right. \\
& \left.-E_{2}+\lambda-\eta\right)-Y_{2}\left(E_{1}-\lambda-\eta\right) Y_{2}\left(E_{2}-\lambda-\eta\right) \\
& +Y_{2}\left(\eta, E_{1}-\eta\right)+Y_{2}(\eta, \lambda)+Y_{2}\left(\eta, E_{2}-\lambda\right) \\
& +Y_{2}\left(E_{1}-\eta, \lambda\right)+Y_{2}\left(E_{1}-\eta, E_{2}-\lambda\right) \\
& \left.+Y_{2}\left(\lambda, E_{2}-\lambda\right)-1\right]
\end{aligned}
$$

and using the properties of these functions (see the Appendix) it is easy to show that

$$
Y_{2}^{(2)}\left(E_{1}, E_{2}\right) \sim 0, \quad 0<\left|E_{1}-E_{2}\right| \ll E_{1}+E_{2} .
$$

The levels of integrable systems behave as noncorrelated random variables, as shown by Berry and Tabor [2], and therefore $Y_{k}=0$ for any value of $k$. According to Eq. (26) $Y_{2}^{(2)}=0$, suggesting that two-body systems are integrable, provided that the energy of the levels is high enough.

\section{CONCLUSIONS}

In summary, we have analyzed the energy spectral fluctuations of identical particles moving independently in a chaotic mean field. To this purpose we have used matrix en- 
sembles with different statistical properties to model the single-particle energies; then large and complete sequences of energy levels have been generated for boson and fermion systems with different number of particles. The statistical analysis has been performed by means of the nearest neighbor spacing distribution $P(s)$, the $\Delta_{3}(L)$, and the $\delta_{n}$ statistics.

Although there is no extended numerical experience or a rigorous derivation, it is usually assumed that these systems always exhibit Poisson fluctuations. The reason has to be found in a heuristic argument introduced by Bloch several decades ago. It assumes that two successive energy levels of the many-body spectrum are sums of energies of very different parts of the SP spectrum and therefore their variations will not be correlated. Bloch's argument is obvious if the MF is integrable and all its eigenvalues behave as uncorrelated random variables. However, it is not clear whether this assumption is valid in the low energy domain when the MF is chaotic. For this reason, we have only considered SP spectra with RMT-like fluctuations emphasizing the evolution of the spectral fluctuations of the MB system with the excitation energy.

When the low energy domain of the spectrum is not included in the analysis, the spectral fluctuations of NIP systems are those of generic integrable systems, regardless of the number of particles. Therefore, there is a sharp transition with the number of particles $N$; even if the SP spectrum is chaotic, the $N=2$ system already behaves as an integrable system. Only long-range correlations show certain reminiscences of the MF fluctuations at very large energy scales.

On the contrary, our analysis shows that NIP systems do not obey the Poisson statistics at low energy. With regard to short-range correlations, $P(s)$ evolves smoothly from the Wigner distribution to the Poisson distribution as the energy increases. Using the $\delta_{n}$ statistic, we have also found that at each energy there exists a critical energy scale $L_{c}$. Beyond this scale the long-range correlation structure of the spectrum deviates from the Poisson predictions. Moreover, for a given energy $E, L_{c}$ increases with the number of particles; and for a fixed number of particles $N, L_{c}$ increases with energy.

Another important result is that the spectral fluctuations of fermion and boson systems are very similar, at least for these systems where the number of SP levels is much larger than the number of particles.

These results are consistent with the theoretical prediction for the two-level cluster function for $N=2$. We have shown that $Y_{2}^{(2)}\left(E_{1}, E_{2}\right)$ goes to zero when the energy is high enough.

\section{ACKNOWLEDGMENTS}

We are particularly indebted to P. A. Mello for enlightening discussions. We also thank K. Wood for his help with this manuscript. This work is supported in part by Spanish Government Grants No. BFM2003-04147 and No. FTN200308337-C04-04.

\section{APPENDIX}

Here we derive explicit expressions or obtain bounds for the four types of integrals appearing in Eq. (25). The first is

$$
I_{1}=\frac{1}{E_{1} E_{2}} \int_{0}^{E_{1}} d \eta \int_{0}^{E_{2}} d \lambda Y_{4}\left(\eta, E_{1}-\eta, \lambda, E_{2}-\lambda\right)
$$

Here we use the fact that the two-point cluster functions go quickly to zero when their arguments increase, that is, the arguments should be sufficiently small in order to have significant contributions to the integral,

$$
\begin{gathered}
|\eta-\lambda| \leqslant \varepsilon, \quad\left|E_{1}-E_{2}+\lambda-\eta\right| \simeq|\lambda-\eta| \leqslant \varepsilon, \\
\left|E_{1}-\lambda-\eta\right| \leqslant \varepsilon, \quad\left|E_{2}-\lambda-\eta\right| \leqslant \varepsilon, \\
\left|E_{1}-2 \eta\right| \leqslant \varepsilon, \quad\left|E_{2}-2 \lambda\right| \leqslant \varepsilon,
\end{gathered}
$$

where $\varepsilon$ is a sufficiently small amount of energy ( $\varepsilon$ $\left.\ll E_{1}, E_{2}\right)$ and we have used the fact that $E_{1}$ and $E_{2}$ are close enough $\left(\left|E_{1}-E_{2}\right| \ll \varepsilon\right)$. Now we can write

$$
I_{1} \simeq \frac{1}{E_{1} E_{2}} \int_{E_{1} / 2-\varepsilon / 2}^{E_{1} / 2+\varepsilon / 2} d \eta \int_{E_{2} / 2-\varepsilon / 2}^{E_{2} / 2+\varepsilon / 2} d \lambda Y_{4}\left(\eta, E_{1}-\eta, \lambda, E_{2}-\lambda\right) .
$$

Taking into account the property of the $k$-level cluster functions $\left|Y_{k}\left(E_{1}, E_{2}, \ldots, E_{k}\right)\right|<k$ ! we have

$$
\begin{aligned}
I_{1} & \leqslant \frac{1}{E_{1} E_{2}} \int_{E_{1} / 2-\varepsilon / 2}^{E_{1} / 2+\varepsilon / 2} d \eta \int_{E_{2} / 2-\varepsilon / 2}^{E_{2} / 2+\varepsilon / 2} d \lambda\left|Y_{4}\left(\eta, E_{1}-\eta, \lambda, E_{2}-\lambda\right)\right| \\
& \leqslant 3 ! \frac{\varepsilon^{2}}{E_{1} E_{2}}
\end{aligned}
$$

The second integral is

$$
I_{2}=\frac{1}{E_{1} E_{2}} \int_{0}^{E_{1}} d \eta \int_{0}^{E_{2}} d \lambda Y_{3}\left(E_{1}-\eta, \lambda, E_{2}-\lambda\right) .
$$

Now, using that

$$
Y_{3}\left(E_{1}-\eta, \lambda, E_{2}-\lambda\right)=Y_{3}\left(0, E_{1}-\eta-\lambda, E_{2}-2 \lambda\right)
$$

and the change of variables $\alpha=E_{2}-2 \lambda$ and $\beta=E_{1}-\eta-\lambda$ we get

$$
I_{2}=\frac{1}{2 E_{1} E_{2}} \int_{-E_{2}}^{E_{2}} d \alpha \int_{\alpha / 2-E_{2} / 2}^{E_{1}-E_{2} / 2+\alpha / 2} d \beta Y_{3}(0, \beta, \alpha),
$$

and the domain of integration here approaches $\mathbb{R}^{2}$ as $E_{1}$ and $E_{2}$ grow. Thus, making use of the relation between consecutive $Y_{k}$ functions valid for the three classical ensembles and their interpolations (see Ref. [34])

$$
\int_{-\infty}^{\infty} Y_{n}\left(x_{1}, \ldots, x_{n}\right) d x_{n}=(n-1) Y_{n-1}\left(x_{1}, \ldots, x_{n-1}\right)
$$

we arrive at

$$
I_{2} \simeq \frac{1}{E_{1} E_{2}}
$$

The third type is 


$$
I_{3}=\frac{1}{E_{1} E_{2}} \int_{0}^{E_{1}} d \eta \int_{0}^{E_{2}} d \lambda Y_{2}\left(2 \eta-E_{1}\right) Y_{2}\left(2 \lambda-E_{2}\right)
$$

Using the change of variables $\alpha=2 \eta-E_{1}$ and $\beta=2 \lambda-E_{2}$

$$
\begin{aligned}
I_{3} & =\frac{1}{4 E_{1} E_{2}} \int_{-E_{1}}^{E_{1}} d \alpha \int_{-E_{2}}^{E_{2}} d \beta Y_{2}(\alpha) Y_{2}(\beta) \\
& =\frac{1}{4 E_{1} E_{2}}\left[1+O\left(\frac{1}{E_{1}}\right)\right]\left[1+O\left(\frac{1}{E_{2}}\right)\right],
\end{aligned}
$$

where in the last equation we have made use of the known integrals of the two-point cluster functions of the random matrix ensembles [26].

The fourth integral is

$$
\begin{aligned}
I_{4} & =\frac{1}{E_{1} E_{2}} \int_{0}^{E_{1}} d \eta \int_{0}^{E_{2}} d \lambda Y_{2}\left(2 \eta-E_{1}\right) \\
& =\frac{1}{E_{1}} \int_{0}^{E_{1}} d \eta Y_{2}\left(2 \eta-E_{1}\right)
\end{aligned}
$$

and using the change of variables $\mu=2 \eta-E_{1}$

$$
\begin{aligned}
I_{4} & =\frac{1}{2 E_{1}} \int_{-E_{1}}^{E_{1}} Y_{2}(\mu) d \mu=\frac{1}{E_{1}} \int_{0}^{E_{1}} Y_{2}(\mu) d \mu \\
& =\frac{1}{E_{1}}\left[\frac{1}{2}-\frac{1}{\nu \pi^{2} E_{1}}+O\left(\frac{1}{E_{1}^{2}}\right)\right],
\end{aligned}
$$

where $\nu$ is the repulsion parameter. As we can see, all these four integrals go to zero in the high-energy limit.
[1] H.-J. Stöckmann, Quantum Chaos (Cambridge University Press, Cambridge, UK, 1999).

[2] M. V. Berry and M. Tabor, Proc. R. Soc. London, Ser. A 356, 375 (1977).

[3] O. Bohigas, M. J. Giannoni, and C. Schmit, Phys. Rev. Lett. 52, 1 (1984).

[4] G. Casati, F. Valz-Gris, and I. Guarneri, Lett. Nuovo Cimento Soc. Ital. Fis. 28, 279 (1980).

[5] C. Bloch, in Physique Nucléaire, edited by C. DeWitt and V. Gillet (Gordon and Breach, New York, 1969), p. 303.

[6] V. K. B. Kota, Phys. Rep. 347, 223 (2001).

[7] H. A. Weidenmüller, Phys. Rev. A 48, 1819 (1993).

[8] J. Sakhr and N. D. Whelan, Phys. Rev. E 67, 066213 (2003).

[9] G. Tanner, K. Richter, and J.-M. Rost, Rev. Mod. Phys. 72, 497 (2000).

[10] J. Sakhr and N. D. Whelan, Phys. Rev. A 62, 042109 (2000).

[11] T. A. Brody, J. Flores, J. B. French, P. A. Mello, A. Pandey, and S. S. M. Wong, Rev. Mod. Phys. 53, 385 (1981).

[12] M. Srednicki, Phys. Rev. E 66, 046138 (2002).

[13] L. Benet, T. Rupp, and H. A. Weidenmüller, Ann. Phys. (N.Y.) 292, 67 (2001).

[14] J. B. French and S. S. M. Wong, Phys. Lett. 33B, 449 (1970).

[15] The concept of integrability in quantum mechanics is very subtle and deserves some attention. Various attempts to give a unique and precise definition of integrability in quantum mechanics have not produced irrefutable answers (see, for instance, [16-18] and references therein). Generally a quantum system is said to be integrable if a set of as many commuting Hermitian operators as quantum degrees of freedom can be explicitly given, and the Hamiltonian can be expressed as a function of these operators $[17,19]$. This criterion, relying on the algebraic structure of quantum mechanics, does not directly refer to classical mechanics, and is closely related to exact solvability.

[16] St. Weigert, Physica D 56, 107 (1992).

[17] S. Weigert and G. Muller, Chaos, Solitons, and Fractals 5, 1419 (1995).

[18] W. M. Zhang and D. H. Feng, Phys. Rep. 252, 1 (1995).
[19] A. Relaño, J. Dukelsky, J. M. G. Gómez, and J. Retamosa, Phys. Rev. E 70, 026208 (2004).

[20] J. R. Huzenga and L. G. Moretto, Annu. Rev. Nucl. Sci. 22, 427 (1972).

[21] P. Leboeuf, A. G. Monastra, and A. Relaño, Phys. Rev. Lett. 94, 102502 (2005).

[22] H. A. Bethe, Rev. Mod. Phys. 9, 69 (1937); H. A. Bethe, Phys. Rev. 50, 332 (1936).

[23] T. Ericson, Nucl. Phys. 11, 481 (1959); A. G. W. Cameron, Can. J. Phys. 36, 1040 (1958).

[24] Handbook of Mathematical Formulas, edited by M. Abramowitz and I. A. Stegun (Dover Publications, New York, 1972).

[25] R. E. Walpole, R. H. Myers, and S. L. Myers, Probability and Statistics for Engineers and Scientists, 6th ed. (Prentice-Hall, Englewood Cliffs, NJ, 2001).

[26] M. L. Mehta, Random Matrices (Academic Press, New York, 1991).

[27] A. Relaño, J. M. G. Gómez, R. A. Molina, J. Retamosa, and E. Faleiro, Phys. Rev. Lett. 89, 244102 (2002).

[28] On one hand, as $L$ increases the number of available intervals to perform the average decreases and the statistical results are less significant. On the other, the calculation of the rigidity is quite sensitive to the unfolding procedure. If the mean level density is not accurately known, the unfolding method will introduce accumulated errors that eventually give rise to a spurious saturation of $\left\langle\Delta_{3}(L)\right\rangle$ for large $L$ values. See, for instance, [29].

[29] J. M. G. Gómez, R. A. Molina, A. Relaño, and J. Retamosa, Phys. Rev. E 66, 036209 (2002).

[30] M. V. Berry and M. Robnik, J. Phys. A 17, 2413 (1984).

[31] E. Faleiro, J. M. G. Gómez, R. A. Molina, L. Muñoz, A. Relaño, and J. Retamosa, Phys. Rev. Lett. 93, 244101 (2004).

[32] J. M. G. Gómez, A. Relaño, J. Retamosa, E. Faleiro, M. Vranicar, and M. Robnik, Phys. Rev. Lett. 94, 084101 (2005).

[33] M. S. Santhanam and J. N. Bandyopadhyay, Phys. Rev. Lett. 95, 114101 (2005).

[34] J. B. French, V. K. B. Kota, A. Pandey, and S. Tomsovic, Ann. Phys. (N.Y.) 181, 198 (1988). 